\title{
Indications for Enhanced Auger-Electron Absorption in a Hot-Electron Gas
}

\author{
G. Schiwietz, ${ }^{*}$ M. Roth, ${ }^{\dagger}$ K. Czerski, ${ }^{\ddagger}$ and F. Staufenbiel ${ }^{\S}$ \\ Hahn-Meitner-Institut, Bereich SF, Glienicker Strasse 100, 14109 Berlin, Germany
}

\section{P. L. Grande}

Instituto de Física, Universidade Federal do Rio Grande do Sul, 91501-970, Porto Alegre, RS, Brazil (Received 21 May 2007; revised manuscript received 29 August 2007; published 9 November 2007)

\begin{abstract}
Solid-state Auger-electron angular distributions are known to be largely independent of the type of excitation, following roughly a cosine law for low ejection energies. In this Letter it is shown that the iontrack dynamics and the corresponding high electron temperatures lead to significant variations of these Auger distributions. Ratios for different degrees of inner-shell ionization versus angle are sensitive to the high-energy-deposition density. The ratios show a minimum for emission angles close to the ion-track direction, consistent with enhanced inelastic electron-energy losses or electron absorption, respectively. Thus Auger-electron yields are influenced by the spatial electronic excitation distribution.
\end{abstract}

Hot electrons play an important role for the heat transport inside highly excited materials by laser or ion bombardment as well as in other areas such as in degenerate cores of white dwarfs and giant stars [1]. In fact, the nonequilibrium electron temperatures determine the charge-state dynamics of ions in the solar wind [2] as well as the dynamics in tokamak plasmas [3]. Furthermore, the influence of the electron temperature on the behavior of clusters, plasmas, solids, and solid-state surfaces excited by high-power short-pulse lasers is the subject of intense research worldwide. There is rapidly growing knowledge not only on the corresponding materials modification [4] but also on the primary electron temperature [5] and on ejected electrons [6] and ions [7]. The trend towards short-pulse x-ray lasers [8] shows that high-power laser beams and laser-solid interaction processes [9] are approaching the time resolution, spatial resolution, and mean energy transfer of individual swift heavy ions interacting with solids. Ions (lasers) transfer $50 \%$ (nearly $100 \%$ ) of their energy via dipole transitions during an interaction time of $10^{-18}$ to $10^{-17} \mathrm{~s}\left(10^{-15}\right.$ to $\left.10^{-13} \mathrm{~s}\right)$ within a nm spot for a single ion (a $\mu \mathrm{m}$ spot for a laser beam of similar power density). Both types of radiation, fast heavy ions and high-power lasers, are complementary laboratory sources and probes of nonequilibrium processes driven by strong electronic excitations.

So far, electron temperatures in ion-solid interactions have been measured using only high-resolution spectroscopy of Auger electrons, resulting from the decay of target inner-shell vacancies in the central ion-track region. Most quantitative determinations are based on the high-energy slopes of Auger structures induced by incident ions in comparison with those resulting from incident electrons as a (virtually asymptotic) low-temperature reference. Using different Auger lines, these data yield snapshots of the time evolution of the solid a few $10^{-15} \mathrm{~s}$ after the rapid electronic excitation. Contrary to most lattice-modification studies, such investigations allow for a clear distinction of track-formation mechanisms [10].

The pathways of the relaxation of high ionization densities inside solids and the formation of the so-called ion tracks have been the subject of a long-standing discussion $[11,12]$. During the past few years, however, it became obvious that the electronic thermal spike (atomic motion due to electron-atom collisions and electron-phonon couplings) plays a major role for the atomic rearrangement processes in metals and semiconductors [10,12]. High electron temperatures, consistent with the populated electronic density of states (e-DOS) near the Fermi level $[10,13,14]$, have been identified as a possible driving force of atomic motion.

In this work, we take advantage of the columnar excitation with nm radius and attosecond timing related to fast heavy ions. We present clear evidence for a significant difference between electron- and fast ion-induced Augerelectron angular distributions, never observed so far. This difference points to an influence of the free $e$-DOS on the transport of fast electrons through the hot-electron gas of the cylindrical ion track, as will be discussed further below.

Experiments have been performed with heavy ions at $8 \%$ the speed of light $\left(592 \mathrm{MeV}{ }^{197} \mathrm{Au}\right)$ and electrons of similar velocity. The RFQ-cyclotron combination of the Hahn-Meitner-Institut has delivered stable $\mathrm{Au}^{q+}$ ion beams during several beam times at the charge state $q=$ $30+$ with electrical currents of about $300 \mathrm{nA}$. The projectile charge-state equilibrium at $q_{\text {mean }}=46.3+$ [15] was obtained using a thin carbon stripper foil about $1 \mathrm{~m}$ upstream from the target. The magnetically shielded ultrahigh vacuum setup is described in detail elsewhere [16], and thus only a brief description shall be given here.

We have used polycrystalline beryllium (Be) and crystalline aluminum $(\mathrm{Al})$ samples, since both targets possess strong low-energy Auger lines with angular distributions not significantly influenced by the atomic structure. In this 
work, we concentrate mainly on the $\mathrm{Al}$ data since $\mathrm{Al}$ is close to a free electron gas, a fact that simplifies theoretical treatments. Moreover, it is possible to compare ion- and electron-induced data as will be discussed further below. Although the fast $\mathrm{Au}$ ions penetrate deep inside the target, Auger electrons at about $100 \mathrm{eV}$ as considered here stem only from the first few surface layers [17]. This has three consequences.

First, the effective target volume is a very thin layer close to the surface and attention has to be paid to the preparation of atomically clean surfaces (vacuum pressure in the low $10^{-10}$ mbar during the experimental runs) using sputtering/heating cycles before the ion irradiation. These surfaces stayed atomically clean during the one to two days of each of the beam times, mainly because of ion-induced desorption of coverage atoms.

Second, there is no depth dependence of ionization within this shallow effective target volume.

Third, electron-collision cascades of fast $\delta$ electrons (secondary electrons generated directly due to the interaction of projectiles with target atoms) close to the surface may produce inner-shell vacancies and contribute indirectly to the Auger signal [18]. An estimate for this unwanted fraction of the Auger signal yields about $25 \%$ for Al $L^{1} V V$ and about $10 \%$ for Be $K^{1} V V$ Auger electrons.

The earth magnetic field is reduced by about a factor of 130 in order to enable precise energy and angle scans with the movable electron spectrometer inside the $\mu$-metal shielding of the chamber [16]. As implicitly assumed in Fig. 1, the manipulator tilt axis was used to adjust the target surface-normal antiparallel to the electron or ion-beam direction, respectively. Thus, all data in this work are given for normal-incidence beams and the beam direction defines the zero of the angular scales for the subsequent figures.

Figure 2 displays the multiline structure of the Auger energy spectrum, for an emission angle of $\Theta_{e}=150^{\circ}$ with respect to the incident Au ion beam. The continuous background due to $\delta$ electrons has already been subtracted. A separation of the different multi-ionization components

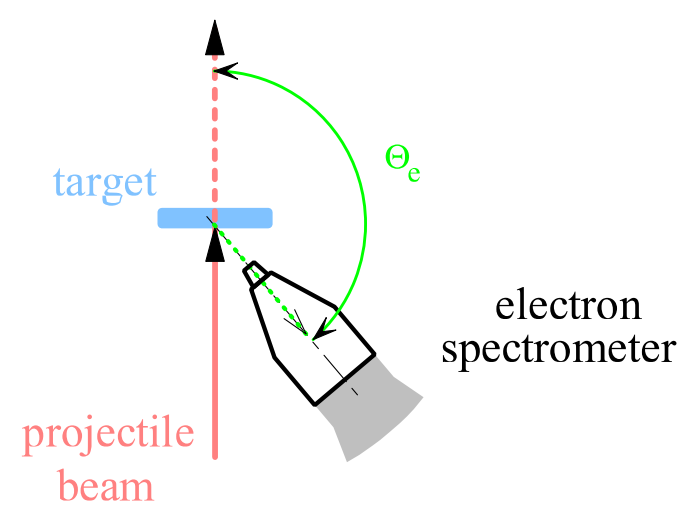

FIG. 1 (color online). Schematic view of the target, projectile beam at normal incidence and electron spectrometer.
$L^{n} V V$ for $n=1$ to 5 aluminum $L$-shell vacancies has been performed iteratively, consistent with previous assignments $[10,19]$. The weak $L^{5} V V$ Auger structure at about $130 \mathrm{eV}$ is just beyond the limit of being statistically significant.

Note that electron-induced Auger spectra (not shown here) consist mainly of the $L^{1} V V$ diagram line plus a small $L^{2} V V$ satellite component due to shakeoff processes. Comparison with ion-induced spectra for the semimetal $\mathrm{Be}$ and the conductor $\mathrm{Al}$ reveals the following two facts. No significant Auger-electron energy reductions have been found for incident ions corresponding to a rapid neutralization of the ion-track potential created by ionized target atoms $[10,13]$. Furthermore, electron temperatures of up to $100000 \mathrm{~K}$ for individual Au ions have been extracted by comparison of the broadened ion-induced Auger lines with the corresponding narrower line shapes obtained for incident electrons [13].

Figure 3 displays the ratio of Auger-line intensities $\left[R_{2}(\mathrm{Al})\right.$ and $\left.R_{3}(\mathrm{Al})\right]$ for $\mathrm{Al} L^{n} V V$ with $n=2$ (squares) and 3 (circles) divided by the $\mathrm{Al} L^{1} V V$ value versus emission angle. Fixed energy regions (with a width of about $\pm 10 \mathrm{eV}$ ) centered at the peak maxima in Fig. 2 have been accounted for in the peak-integration procedure. This treatment is largely independent of the quality of the background subtraction, of the peak separation, and of the peak position (the peak maxima are constant to within $\pm 0.2 \mathrm{eV}$ ). Further, it also reduces the statistical uncertainties. The background subtraction, however, is still the main source of uncertainty, especially for the small peaks $\left(L^{3} V V\right.$ for incident ions and $L^{2} V V$ for incident electrons) and dominates the error bars in Fig. 3. Previously published preliminary values for $\mathrm{Be}$ [13] have shown similar lineintensity ratios $R_{2}(\mathrm{Be})$ for the $K^{2} V V$ and $K^{1} V V$ transitions (a reevaluation with slightly modified fit functions has confirmed the preliminary Be results).

It is seen that the ion-induced Auger ratios in Fig. 3 (solid symbols) are steadily decreasing with increasing detection angle. This behavior is in clear contrast to the

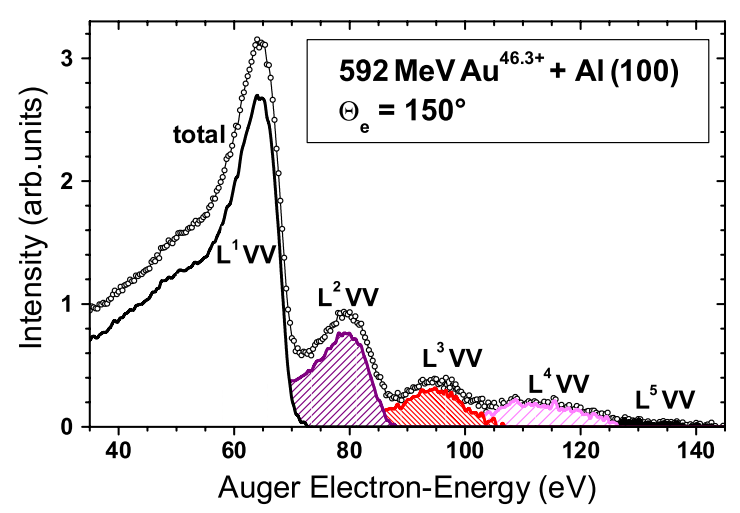

FIG. 2 (color online). Decomposed Al Auger-electron spectrum determined for an angle of $150^{\circ}$. The separated peak structures represent different degrees of $L$-shell ionization. 


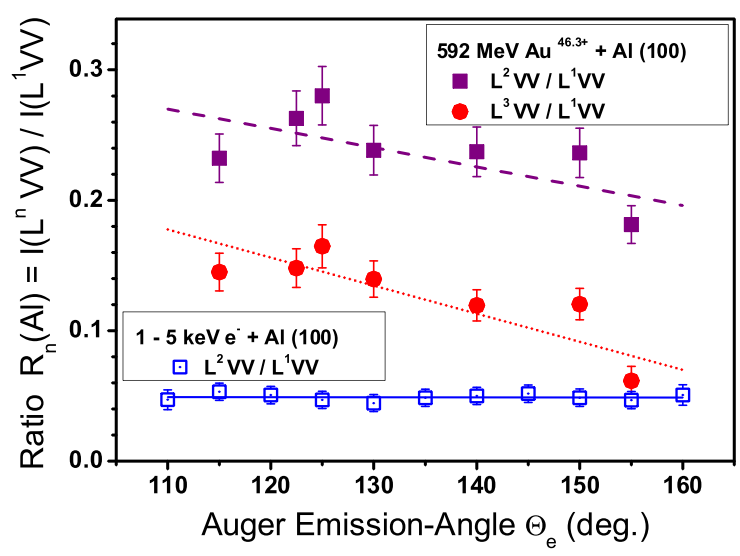

FIG. 3 (color online). Angular distribution of Al Augerelectron line-intensity ratios for some $L^{n} V V$ transitions (see text). The straight lines represent error-weighted linear fits.

typical angular distribution of slow Auger electrons that is approximately described by a cosine function $[18,20,21]$ and thus by a constant value of the ratio. Based on a ballistic Auger-electron model $[18,20]$ this behavior is shown in Eq. (1) for the energy and angular distribution of ejected electrons, resulting from a $\delta$-like initial Augerelectron energy distribution. The following doubly differential emission yield $\frac{d^{2} P_{A}}{d \Omega_{e} d E_{e}}$ as a function of the final ejection angle $\Theta_{e}$ and the measured electron energy $E_{e}$ is valid for those energies, where diffusion does not influence the electron transport from a distributed isotropic source to the surface:

$$
\frac{d^{2} P_{A}}{d \Omega_{e} d E_{e}}\left(\Theta_{e}, E_{e}\right)=\frac{1}{4 \pi}\left|\frac{\cos \Theta_{e}}{S_{e}\left(E_{e}\right)}\right| \rho \sigma_{A}^{\text {eff }} .
$$

$S_{e}\left(E_{e}\right)$ is the specific electronic energy loss for electrons in a medium characterized by the atomic density $\rho$ and by the effective Auger-electron emission cross section $\sigma_{A}^{\text {eff }}$. The cross section $\sigma_{A}^{\text {eff }}$ includes direct ionization processes by the projectile, (indirect) electron-collision cascades [18] and the Auger-electron decay yield [22]. It is seen that Eq. (1) follows strictly a cosine law. Exact cosine dependences do not only result from the path length of ballistic electron transport in homogenous media, but also due to refraction of low-energy electrons at a planar surface potential [23]. However, small deviations from the cosine distribution have been reported for various noncrystalline samples involving different types of transitions, and they are assigned to surface excitations by the escaping electrons [21]. These deviations might explain a few percent decrease in the ratio, but not the steep decrease corresponding to values of $-25 \%$ and $-55 \%$ for the two $\mathrm{Al}$ line ratios and $-15 \%$ for the $\mathrm{Be}$ ratio (corresponding to $\Theta_{e}=115^{\circ}$ and $160^{\circ}$ ).

In this work, we have concentrated on ratios, in order to largely cancel possible influences of diffraction effects, nonplanar surface morphologies, or other experimental uncertainties. However, a remaining deviation from the constant ratio might in principle be due to an Augerelectron energy dependence of the yield versus $\Theta_{e}$. But this can be checked experimentally, by using the diagram and the shakeoff lines of $\mathrm{Al}$ both induced by normally incident electrons. The results of these measurements are plotted in Fig. 3 for the $R_{2}(\mathrm{Al})$ ratio as open squares. They are consistent with a nearly constant ratio, in contrast to the same ratio for heavy ions (closed squares). Thus, the decreasing intensity observed for the heavy ions seems to reflect an effect of the type of projectile and not the target structure or the Auger-electron decay process. A possible explanation is given in the following discussion.

It is known [10,24-26] that fast electrons and fast ions transfer their energy to the target in similar ways. Ionization probabilities, however, scale for (nearly) bare particles roughly with $\left(q / v_{p}\right)^{2}$, where $q$ is the projectile charge state and $v_{p}$ is the projectile velocity. Hence, in our case the ionization probabilities for heavy ions exceed the electron-induced ones by a factor of about 2000, resulting in a strongly enhanced inner-shell ionization and valenceelectron temperature (the ionization track) [13]. This highenergy density is even more pronounced for lines due to multiple inner-shell ionization in comparison to the diagram lines. The corresponding vacancies are localized closer to the track center and, even more important, they decay faster, being related to a higher temperature in the ion track [13]. Hence, the reduced Auger-electron ratio close to angles of $\Theta_{e}=180^{\circ}$ in Fig. 3 may be understood if there is enhanced electron absorption for fast electrons that move along the track axis (the surface-normal direction) during its maximum temperature stage.

Consequently, the largest absorption should be found for the highest temperature of about $50000 \mathrm{~K}$ corresponding to the Al $L^{3} V V$ transition, which is in agreement with our experimental observation of a steeper slope for $R_{3}(\mathrm{Al})$ in comparison to $R_{2}(\mathrm{Al})$. Theoretically, one expects the collective dynamic screening of the Auger electron to dominate the interaction at low speeds, leading to reduced energy losses at elevated temperatures [27]. For Auger electrons far above the Fermi velocity, however, screening should be less important, and the variation of the density of states near the Fermi level opens new excitation channels, which should give rise to enhanced absorption as indicated by our results. In other words, our data indicate that $S_{e}\left(E_{e}\right)$ in Eq. (1) should for heavy ions be replaced by a $\Theta_{e}$-dependent averaged energy loss $S_{e}^{\text {mean }}\left(E_{e}, \Theta_{e}, T_{e}(r)\right)$, where $T_{e}(r)$ is the radial electronic temperature field around the ion path and during the Auger-electron decay. In addition, we expect $S_{e}^{\text {mean }}$ to increase for electronemission angles $\Theta_{e}$ close to $180^{\circ}$ and for high electron temperatures $T_{e}$.

In conclusion, a new ion-track effect, namely, a shorttime modification of the Auger-electron angular distribution, has been proven by comparison of ion- and electron- 
induced yields. The measured intensity variation is consistent with enhanced absorption of fast electrons that move for an extended time close to the hot center of the heavy-ion track. Future theoretical and experimental studies might reveal that quantitative temperature gradients at nanometer dimensions and on a femtosecond time scale can be extracted from such data.

This work was partially supported by the CNPq and CAPES agencies, the Humboldt Foundation, and the PROBRAL cooperation program (DAAD and CAPES). We also thank the ISL accelerator crew for delivering the necessary high quality ion beams.

*schiwietz(at)hmi.de

http://www.hmi.de/people/schiwietz/index_e.html

'Present address: OSRAM GmbH, AM D-B, Nonnendammallee 44, D-13629 Berlin, Germany.

Present address: Institute of Physics, University of Szczecin, ul. Wielkopolska 15, 70-451 Szczecin, Poland.

${ }^{\S}$ Present address: Forschungszentrum Dresden-Rossendorf, Abt. Strahlungsquelle ELBE, Bautzner Landstr. 128, 01328 Dresden, Germany.

[1] P.S. Shternin and D. G. Yakovlev, Phys. Rev. D 74, 043004 (2006).

[2] Yuan-Kuen Ko, Lennard A. Fisk, Johannes Geiss, George Gloeckler, and Madhulika Guhathakurta, Sol. Phys. 171, 345 (1997).

[3] Y. Hamada, T. Watari, A. Nishizawa, K. Narihara, Y. Kawasumi, T. Ido, M. Kojima, and K. Toi, Phys. Rev. Lett. 96, 115003 (2006).

[4] M. Bonn, S. Funk, Ch. Hess, D. N. Denzler, C. Stampfl, M. Scheffler, M. Wolf, and G. Ertl, Science 285, 1042 (1999).

[5] J. G. Fujimoto, J. M. Liu, E. P. Ippen, and N. Bloembergen, Phys. Rev. Lett. 53, 1837 (1984).

[6] M. Tatarakis, F. N. Beg, E. L. Clark, A. E. Dangor, R. D. Edwards, R. G. Evans, T. J. Goldsack, K. W. D. Ledingham, P. A. Norreys, M. A. Sinclair, M.-S. Wei, M. Zepf, and K. Krushelnick, Phys. Rev. Lett. 90, 175001 (2003).

[7] T. Ditmire, J. W. G. Tisch, E. Springate, M. B. Mason, N. Hay, R. A. Smith, J. Marangos, and M.H.R. Hutchinson, Nature (London) 386, 54 (1997).

[8] R. Neutze, R. Wouts, D. van der Spoel, E. Weckert, and J. Hajdu, Nature (London) 406, 752 (2000).

[9] T. Laarmann, H. Wabnitz, J. Schulz, A. R. B. de Castro, P. Gürtler, W. Laasch, and T. Möller, Phys. Rev. Lett. 95, 063402 (2005).
[10] G. Schiwietz, M. Roth, K. Czerski, F. Staufenbiel, and P. L. Grande, Nucl. Instrum. Methods Phys. Res., Sect. B 225, 4 (2004); 226, 683 (2004).

[11] R. L. Fleischer, P. B. Price, and R. M. Walker, Nuclear Tracks in Solids (University of California Press, Berkeley, CA, 1975).

[12] Z. G. Wang, C. Dufour, E. Paumier, and M. Toulemonde, J. Phys. Condens. Matter 6, 6733 (1994).

[13] G. Schiwietz, G. Xiao, P. L. Grande, E. Luderer, R. Pazirandeh, and U. Stettner, Europhys. Lett. 47, 384 (1999); F. Staufenbiel, G. Schiwietz, K. Czerski, M. Roth, and P. L. Grande, Nucl. Instrum. Methods Phys. Res., Sect. B 230, 426 (2005).

[14] M. Caron, H. Rothard, M. Beuve, and B. Gervais, Phys. Scr. T92, 281 (2001).

[15] Details of the charge-state data for Au in C are currently being prepared for publication.

[16] G. Schiwietz, K. Czerski, M. Roth, F. Staufenbiel, E. Luderer, and P. L. Grande, Nucl. Instrum. Methods Phys. Res., Sect. B 193, 705 (2002).

[17] C. J. Powell and A. Jablonski, NIST standard reference database 71 version 1.1, National Institute of Standards and Technology, USA (2001).

[18] G. Schiwietz, D. Schneider, J. P. Biersack, N. Stolterfoht, D. Fink, A. Mattis, B. Skogvall, H. Altevogt, V. Montemayor, and U. Stettner, Phys. Rev. Lett. 61, 2677 (1988); G. Schiwietz and G. Xiao, Nucl. Instrum. Methods Phys. Res., Sect. B 107, 113 (1996).

[19] W. Schmidt, P. Müller, V. Brückner, F. Löffler, G. Saemann-Ischenko, and W. Schubert, Phys. Rev. A 24, 2420 (1981).

[20] S. Tougaard and P. Sigmund, Phys. Rev. B 25, 4452 (1982).

[21] W. S. M. Werner, H. Tratnik, J. Brenner, and H. Störi, Surf. Sci. 495, 107 (2001)

[22] M. Krause, J. Phys. Chem. Ref. Data 8, 507 (1979).

[23] M. Rösler and W. Brauer, in Particle Induced Electron Emission I, Springer Tracts of Modern Physics Vol. 122 (Springer, Berlin, 1991).

[24] H. Bethe, Ann. Phys. (Leipzig) 397, 325 (1930).

[25] P. L. Grande and G. Schiwietz, in Advances in Quantum Chemistry, edited by J. Sabin (Elsevier Inc., New York, 2004), Vol. 45, pp. 7-46.

[26] P. Sigmund, Nucl. Instrum. Methods Phys. Res., Sect. B 135, 1 (1998); A. Schinner and P. Sigmund, Nucl. Instrum. Methods Phys. Res., Sect. B 195, 64 (2002).

[27] I. Nagy, A. Arnau, P. M. Echenique, and K. Ladányi, Phys. Rev. A 43, 6038 (1991). 